\title{
Low-Spatial Coherence High-Power Electrically Injected 6xx nm Dual-Emitter Laser
}

\author{
Yufei Jia1,2,3, Linhai Xu1,2,3, Yuzhe Lin'1,2,3, Yufei Wang1,2, Aiyi Qi1,2, Wanhua Zheng1,2,3,4* \\ ${ }^{1}$ State Key Laboratory on Integrated Optoelectronics, Institute of Semiconductors, CAS, Beijing, China \\ ${ }^{2}$ Laboratory of Solid State Optoelectronics Information Technology, Institute of Semiconductors, CAS, Beijing, China \\ ${ }^{3}$ College of Materials Science and Opto-Electronic Technology, University of Chinese Academy of Sciences, Beijing, China \\ ${ }^{4}$ College of Future Technology, University of Chinese Academy of Sciences, Beijing, China \\ Email: *whzheng@semi.ac.cn
}

How to cite this paper: Jia, Y.F., Xu, L.H., Lin, Y.Z., Wang, Y.F., Qi, A.Y. and Zheng, W.H. (2018) Low-Spatial Coherence High-Power Electrically Injected 6xx $\mathrm{nm}$ Dual-Emitter Laser. Journal of Applied Mathematics and Physics, 6, 2338-2342. https://doi.org/10.4236/jamp.2018.611195

Received: October 30, 2018

Accepted: November 19, 2018

Published: November 22, 2018

\begin{abstract}
Laser-based displays have attracted much attention owing to large-size screen and full-color gamut compared with other displays such as liquid crystal display and light emitting diode. However, there exists a phenomenon, speckle, limits the applications of laser display because of the high coherence of laser. In this work, we developed an electrically injected 6xx nm dual-emitter laser which combines the low-spatial coherence with the high-power. The output power of the dual-emitter laser exceeds $500 \mathrm{~mW}$ under $20^{\circ} \mathrm{C}$ pulse operation. The single emitter consists of D-shaped section used to obtain more independent spatial modes thus reduces coherence and a stripe area to obtain the high power. The radius of the D-shaped cavity is $500 \mu \mathrm{m}$ and the length of stripe is $1000 \mu \mathrm{m}$. We used the standard photolithography and inductively coupled plasma (ICP) process to fabricate the device. The speckle contrast was measured to be $5 \%$. It exhibits a great potential of reducing speckle from the source directly for laser display.
\end{abstract}

\section{Keywords}

Low-Spatial Coherence, Dual-Emitter, Red-Emitting Lasers, Speckle Contrast, Laser Display

\section{Introduction}

Laser-based displays have attracted much attention and a bright prospect, because the laser is the only light source that produces saturated primary colors that reach over $90 \%$ of what our eyes can see, in another word, big gamut [1] and the laser display can realize the larger screen. However, there still exist the 
drawbacks such as the speckle, a pattern formed by the interference of the laser owing to the high spatial coherence. The speckle pattern is considered as a negative factor in the laser display [2].During the years, different ways have been presented to reduce the speckle pattern. For instance, angle diversity, polarization diversity, wavelength diversity [3], rotating diffuser [4], and the Grating Light Valve (GLV) [3]. Goodman has proved that the speckle contrast $\mathrm{C}$ will be $\mathrm{N}^{-1 / 2}$ which $\mathrm{N}$ means the independent modes [5]. Nevertheless, the methods mentioned above achieve the low speckle contrast by averaging the speckle patterns in the external cavity. To decrease the speckle effect fundamentally, we have to realize the low-spatial coherence of the light source.

There are some ways to suppress the speckle by reducing the spatial coherence of the laser such as the random laser that can tune the spatial coherence by varying the density of the scatters [6], degenerate cavity which can obtain the many transverse modes to reduce the spatial coherence [7]. However, the random laser and degenerate cavity cannot realize the electrical injection and the compact size. In order to realize the commercialization, there are some conditions to satisfy for the device: electrically injection; high conversion efficiency and directionally emission beam [8]. We recently demonstrated that the electrically injected random lasing based on disordered photonic crystal structures [9] and the low-spatial coherence electrically injected red-emitting semiconductor laser [10].

In this paper, we will present latest results of new low-spatial coherence $6 \mathrm{xx}$ $\mathrm{nm}$ dual-emitter for relative high power. The maximal output power is $510 \mathrm{~mW}$ under $20^{\circ} \mathrm{C}$, pulsewidth of $40 \mu$ s and repetition frequency of $40 \mathrm{~Hz}$ after packaging. The speckle contrast is measured to be $5 \%$ in the same way in [11].

\section{The Structure Design of Device}

We use the coupled-cavity structure that consists the D-shaped cavity and the Fabry Perot (FP) cavity to obtain the multimode lasing simultaneously and relative high power output. The D-shaped cavity structure depends on the R, $d$ and $\mathrm{L}$ which represent the radius of the $\mathrm{D}$-shaped cavity, the distance between the flat edge and the center of the D-shaped cavity and FP cavity length respectively. Figure 1 is the schematic structure of the dual-emitter laser. We simulated the mode distributions of different $\mathrm{d}$ at the wavelength of $630 \mathrm{~nm}$ and adopted $\mathrm{d}=$ $0.5 \mathrm{R}$ because of the uniform mode distribution while the other parameter like $\mathrm{d}$ $=0.8 \mathrm{R}$ showed the stable orbit in the $\mathrm{D}$-shaped cavity. The former has more modes lasing simultaneously.

\section{Device Fabrication}

We adopted the $\mathrm{R}=500 \mu \mathrm{m}, \mathrm{d}=250 \mu \mathrm{m}$ and $\mathrm{L}=1000 \mu \mathrm{m}$ to obtain the more lasing modes in order for low-spatial coherence. The fabrication process is relatively simple and low-cost. We used the standard photolithography and ICP to obtain the pattern of the cavity. The cleaved laser bars coated with $8 \%$ antireflection film to increase output power. Finally, the devices were mounted P-side up 
on $\mathrm{Cu}$ mounts.

\section{Device Result}

\subsection{Power and Spectrum Measurement}

First, the power-current curve is measured first to confirm the maximal power the dual-emitter can reach under $20^{\circ} \mathrm{C}$, pulsewidth of $40 \mu$ s and repetition frequency of $40 \mathrm{~Hz}$. The power of the dual-emitter laser emitted exceeds $500 \mathrm{~mW}$ while the single emitter emits $300 \mathrm{~mW}$. Then we measure the spectrum of the dual-emitter laser at different currents under the same pulse condition. Figure 2 is the spectrum and spectrum width at full-width-at-half-maximum (FWHM). There is an obvious narrowing for spectrum width when the current exceeds the threshold value. It proves the dual-emitter become lasing further.

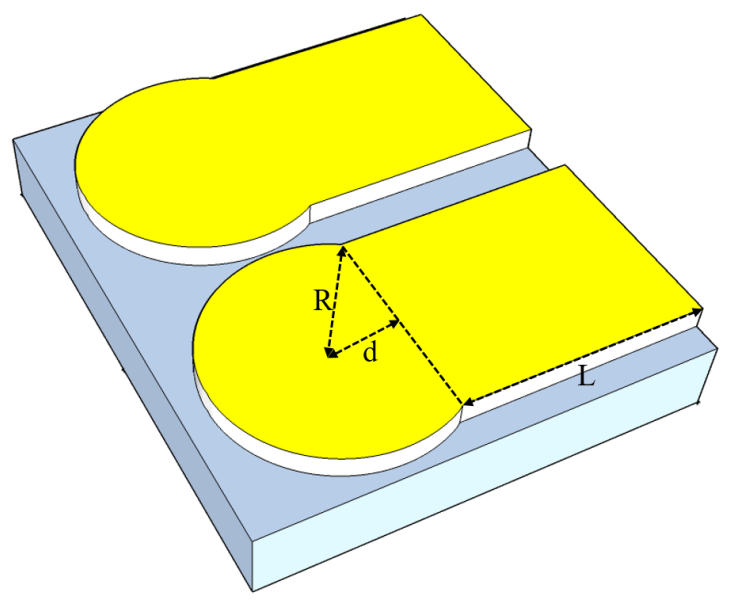

Figure 1. Schematic of the dual-emitter laser.

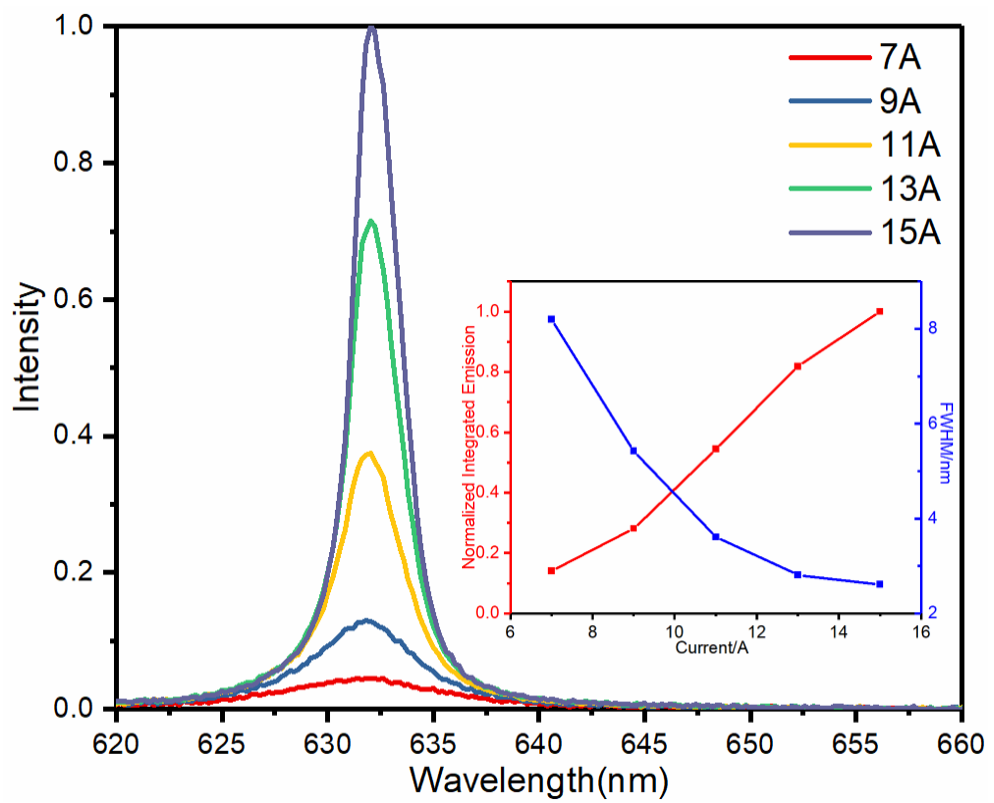

Figure 2. Schematic of the emission spectrum and the spectrum width at FWHM collected from the dual-emitter laser at different currents. 


\subsection{Speckle Measurement}

Next we characterized the spatial coherence of the dual-emitter laser. The speckle contrast $\mathrm{C}$ is introduced to characterize the spatial coherence of the laser. It is defined as:

$$
C=\frac{\sigma_{I}}{\bar{I}} .
$$

The $\sigma_{I}$ is the standard deviation of intensity and $\bar{I}$ is the average intensity. The speckle contrast is a value from 0 to 1 . The low-spatial coherence equivalent to the low $\mathrm{C}$ which means the less influence on the image quality. However, there is still no standard method to measure the speckle [12]. We make a comparison between the dual-emitter laser and the traditional broad area (BA) laser in speckle contrast and image quality. It is similar to the method in [11]. Figure 3 shows the setup of speckle measurement. Figure 4 is the speckle pattern collected from the traditional laser and the dual-emitter laser. We can clearly see the speckle patterns collected from the BA laser and the dual-emitter laser. The speckle contrast of the BA laser is 0.11 while the dual-emitter laser is 0.05 . It means the dual-emitter laser has the lower spatial coherence.

\section{Conclusion}

For the fabrication of the dual-emitter laser, it only needs the standard photolithography and ICP etching process. It shows the low-spatial coherence which

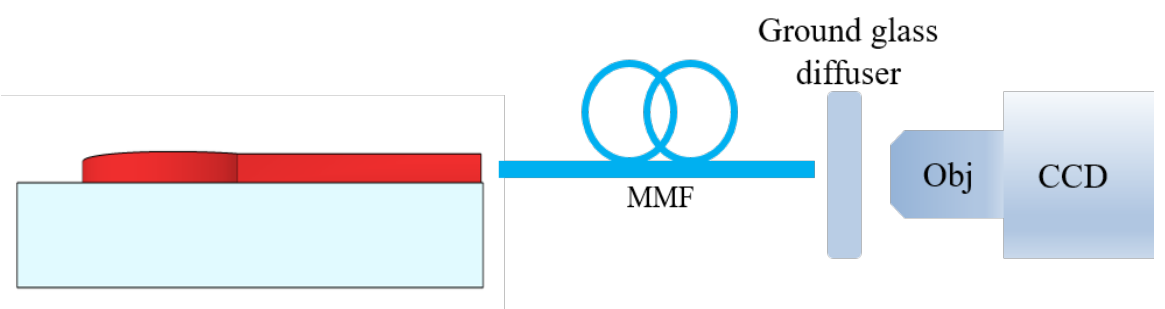

Figure 3. The speckle measurement setup.
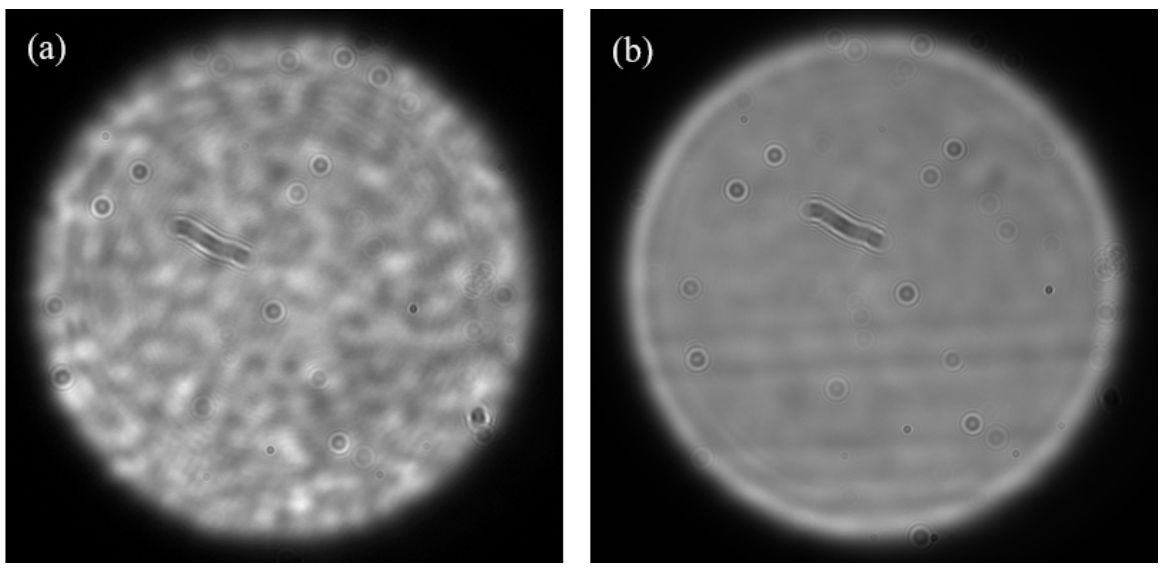

Figure 4. (a) The speckle pattern collected from the BA laser and (b) the dual-emitter laser. 
the speckle contrast is $5 \%$. The relatively power exceeds $500 \mathrm{~mW}$ is obtained under 15A pulse condition ( $40 \mu \mathrm{s} 40 \mathrm{~Hz}$ ). It has a bright prospect as a low-spatial coherence light source in the laser display.

\section{Acknowledgements}

This work was supported by the National Key R \& D Program of China (2016YFB0401804 and 2016YFB0402003).

\section{Conflicts of Interest}

The authors declare no conflicts of interest regarding the publication of this paper.

\section{References}

[1] Jean-Michel (2007) Laser Displays: Life-Like Displays. http://sites.ieee.org/scv-ces/files/2015/06/IEEE_May_22__2007.pdf

[2] Benjamin, D. and Satoshi, K. (1993) Speckle-Free Image in a laser-Diode Microscope by Using the Optical Feedback Effect. Optics Letters, 18, 549-551. https://doi.org/10.1364/OL.18.000549

[3] Jahia, I.T. (2002) Speckle Contrast Reduction in Laser Projection Displays. Proc. SPIE, 4657, 131-137. https://doi.org/10.1117/12.463781

[4] Lowenthal, S. and Joyeux, D. (1971) Speckle Removal by a Slowly Moving Diffuser Associated with Amotionless Diffuser. J. Opt. Soc. Am., 61, 847-851. https://doi.org/10.1364/JOSA.61.000847

[5] Goodman, J.W. (2006) Speckle Phenomena in Optics: Theory and Applications. Version 8.0, Roberts \& Company, Englewood.

[6] Redding, B., Choma, M.A. and Cao, H. (2011) Spatial Coherence of Random Laser Emission. Opt. Lett., 36, 3404-3406. https://doi.org/10.1364/OL.36.003404

[7] Nixon, M., Redding, B., Friesem, A., Cao, H. and Davidson, N. (2013) Efficient Method for Controlling the Spatial Coherence of a Laser. Opt. Lett., 38, 3858-3861. https://doi.org/10.1364/OL.38.003858

[8] Yu, S.F. (2015) Electrically Pumped Random Lasers. J. Phys. D, 48, 483001. https://doi.org/10.1088/0022-3727/48/48/483001

[9] Guo, X.J., Wang, Y.F., Jia, Y.F. and Zheng, W.H. (2017) Electrically-Driven Spectrally-Broadened Random Lasing Based on Disordered Photonic Crystal Structures. Applied Physics Letters, 111, Article ID: 031113. https://doi.org/10.1063/1.4994325

[10] Jia, Y.F., Wang, Y.F., Xu, L.H. and Zheng, W.H. (2018) Low-Spatial Coherence Electrically Pumped Red-Emitting Semiconductor Laser. Proc. SPIE, Beijing, 11-13 October 2018, 10812-10834 (Unpublished).

[11] Redding, B., Cerjan, A., Huang, X., Stone, A.D. and Cao, H. (2015) Low-Spatial Coherence Electrically Pumped Semiconductor Laser for Speckle-Free Full-Field Imaging. PNAS, 112, 1304-1309. https://doi.org/10.1073/pnas.1419672112

[12] Stijn, R., Youri, M., Gordon, C., Guy, V., Peter, J. and Hugo, T. (2012) Standarized Speckle Measurement Method Matched to Human Speckle Perception in Laser Projection Systems. Optics Express, 20, 8770-8783. https://doi.org/10.1364/OE.20.008770 